\title{
2 Physio- und bewegungstherapeutische Aspekte
}

\author{
Christine Graf
}

Die Prävalenz schwerst adipöser Patienten steigt zunehmend und damit auch Komorbiditäten wie arterielle Hypertonie, Typ 2 Diabetes mellitus, etc. Lebensstiländernde Maßnahmen, in diesem Kontext die Bewegungstherapie, spielen eine wichtige Rolle in der Steigerung des Energieverbrauchs und damit der Gewichtsreduktion sowie des Gewichthaltens. Auch in der Planung und nach der Durchführung einer bariatrischen chirurgischen Maßnahme müssen physio- und bewegungstherapeutische Aspekte bedacht werden.

Allerdings werden in den meisten „herkömmlichen“ Bewegungsprogrammen schwer adipöse Patienten aufgrund möglicher Überlastungsschäden am Bewegungsapparat und/ oder Begleiterkrankungen ausgeschlossen. Aus diesem Grund ist wenig bekannt über Möglichkeiten, Machbarkeit und Risiken der Physiotherapie für diese Patienten. Grundsätzlich kann man davon ausgehen, dass körperlich Aktive von der Steigerung des Energieverbrauchs, den metabolischen Effekten, der günstigen Beeinflussung der Körperkomposition, dem Gewichtsverlust und der damit verbundenen Senkung der Belastungen des Muskulo-Skelettal-Systems profitieren. Sie erfahren auch eine Steigerung der Lebensqualität. In präventiven Settings wird ein Umfang von 150 min moderater Aktivität pro Woche empfohlen, bei Adipösen mehr als $300 \mathrm{~min}$.

Voraussetzung für die Teilnahme an einer Bewegungstherapie ist neben der Abklärung und Berücksichtigung möglicher gesundheitlicher Risiken die Beurteilung der individuellen Leistungsfähigkeit, z.B. mittels Gehtest. Daran sollte sich die Ausgestaltung eines Bewegungsprogramms orientieren, ggf. kann anfangs mit physiotherapeutischen Maßnahmen begonnen werden. Ziel ist zunächst eine Stei- gerung der körperlichen Funktionsfähigkeit, um damit eine Unterstützung der Alltagsaktivitäten zu erreichen. Diese (Treppenstegen, zu Fuß gehen, etc.) sollten stets mitempfohlen werden. Zur Überprüfung haben sich Bewegungstagebücher, aber auch der Einsatz von Schrittzählern bewährt. Das Ziel sind 10.000 Schritte pro Tag. Für Patienten mit eingeschränkter Mobilität, z.B. Arthritis, ist Bewegung im warmen Wasser geeignet. Intensive körperliche Aktivität sollte hier vermieden werden, möglicherweise profitieren diese Patienten von Krafttraining. Inwiefern ein Ergometertraining auch im Wasser möglich ist, hängt von den Ausmaßen des Übergewichts $a b$.

Zusammenfassend ist körperliche Aktivität ein wichtiger Teil jeder Adipositastherapie. Allerdings sind die Möglichkeiten bei schwerst Adipösen eher gering, sodass ggf. lediglich physiotherapeutische Maßnahmen zum Erhalt der körperlichen Funktionen eingeleitet werden können.

\subsection{Hintergrund}

In Deutschland ist etwa ein Drittel der Erwachsenen bewegungsinaktiv (Robert Koch-Institut 2005). Dabei ist der gesundheitliche Nutzen von regelmäßiger körperlicher Aktivität heutzutageunumstritten, insbesondere vor dem Hintergrund der Adipositas und möglicher Zusammenhänge mit kardiovaskulären, aber auch metabolischen Risiken. Eine Einteilung von körperlicher Aktivität findet sich in folgender Infobox. 
Klassifikation der körperlichen Aktivität

(mod. nach Pate et al. 1995)

- Metabolische Einheiten oder METs

- Quotienten aus arbeitsmetabolischer Rate zu Ruherate

- entspricht dem Multiplikationsfaktor, um den der Ruhesauerstoffverbrauch von $3,5 \mathrm{mlO}_{2} / \mathrm{kg} / \mathrm{min}$ unter Belastung gesteigert wird.

Beispiel: Für einen $70 \mathrm{~kg}$ schweren Erwachsenen entspricht der Energieverbrauch von einem MET 1,2 kcal/Min.

- leichte Tätigkeiten $=<3$ METS oder $<4 \mathrm{kcal} / \mathrm{Min}$ bzw. weniger als 75 Watt

- moderate Tätigkeiten = 3-6 METS oder 4-7 kcal/Min bzw. 75 bis 100 Watt

- intensive Tätigkeiten $=>6$ METS oder $>7 \mathrm{kcal} / \mathrm{Min}$ bzw. mehr als 100 Watt

Die zugrundeliegenden Mechanismen körperlicher Aktivität sind vielfältig und betreffen neben dem kardiovaskulären System (Ökonomisierung der Herzarbeit) die Steigerung bzw. Verbesserung der Endothelfunktion sowie der NO-Produktion, die Ausschüttung endothelialer Progenitorzellen, aber insbesondere zentrale Signalwege im Bereich des Fettund Kohlenhydratstoffwechsels (zusammengefasst in Graf 2011). So kommt es beispielsweise infolge des mechanischen Reizes der körperlichen Aktivität zu einer Translokation von Glukosetransportern Typ 4 (GLUT4) aus dem Zytoplasma an die Zellmembran und damit zu einer Verbesserung der Insulinresistenz (Berg u. König 2005). Zusätzlich wird die Lipolyse gesteigert, da es infolge eines regelmäßigen Trainings zu einer vermehrten Aktivität entsprechender Enzyme wie der Lecithin-Cholesterol-AcylTransferase (LCAT) und Lipoproteinlipase (LPL) und damit vermittelten Erhöhung hochdichter Lipoproteine, speziell der $\mathrm{HDL}_{2}$-Unterfraktion kommt (Halle et al. 1999). Hinzu tritt eine Reduktion der besonders atherogenen kleinen $\mathrm{LDL}_{5}$ - und $\mathrm{LDL}_{6}$-Unterfraktionen. Letzteres scheint besonders die Folge eines regelmäßigen Ausdauertrainings zu sein. Wenig ist bislang bekannt über den Einfluss von körperlicher Aktivität auf die Adipokine, sicher ist aber, dass im Rahmen der (zentralen) Adipositas körperliche Aktivität eine wesentliche präventive und therapeutische Säule, auch unabhängig von einer Gewichtsabnahme, darstellt. Lee et al. (1999) verfolgten durchschnittlich 8 Jahre lang 21.925 Männer zwischen 3083 Jahren. Die körperliche Leistungsfähigkeit wurde anhand eines maximalen Laufbandtests ermittelt und in Relation zur Körperfettmasse (nicht BMI) gestellt. Unter Berücksichtigung möglicher Einflussfaktoren wie Alter, Nikotin- und Alkoholkonsum und der Familienanamnese wiesen die unfitten, schlanken Männer ein höheres kardiovaskuläres Risiko auf als die fitten adipösen. Vergleichbares beschrieben die Autoren für einen erhöhten Bauchumfang. In einer Folgestudie mit 297 gesunden Männern, bei denen die Körperkomposition anhand Computertomografie oder Magnetresonanztomografie bestimmt wurde, zeigte sich ebenfalls ein geringeres metabolisches Risiko bei gleicher Körperkomposition aber besserer Fitness (Lee et al. 2005).

Diese letzteren Aspekte sind natürlich für die Ausgestaltung einer Therapie bzw. für die Zielsetzung im Rahmen einer interdisziplinären Adipositas-Therapie von großer Bedeutung. Insbesondere morbid Adipöse (BMI > 40) werden jedoch von Bewegungsprogrammen aufgrund möglicher gesundheitlicher Risiken, aber auch Überlastungen und bereits bestehender Schäden sowie Beschwerden im Bereich des muskulo-skelettalen Systems ausgeschlossen.

Da aber die Entstehung von Übergewicht stets die Dysbalance zwischen der Energieaufnahme und dem -verbrauch darstellt, müssen sowohl wissenschaftlich wie auch anwenderorientiert Handlungsempfehlungen zusammengestellt werden, die im ambulanten und stationären Setting umgesetzt werden können, um eine langfristige Lebensstiländerung einzuleiten und die gesundheitlichen Vorteile von körperlicher Aktivität nutzen zu können.

In diesem Kontext muss insbesondere der günstige Einfluss auf die Lebensqualität und dieSteigerung der Alltagstauglichkeit betont werden, die für Betroffene aufgrund ihrer Bewegungseinschränkung häufig vordergründig ist. In diesem Beitrag wird daher neben den allgemeinen Empfehlungen und Begriffsbestimmungen ein maßgeblicher Schwerpunkt auf die Darstellung möglicher Umsetzungen und Anwendungen für morbid Adipöse gelegt.

\subsection{Begriffsbestimmungen und allgemeine Empfehlungen}

Allgemein wird körperliche Aktivität als jede Bewegungsform definiert, die mit einer Steigerung des Energieverbrauchs einhergeht(Caspersenet al. 1985). Körperliche Fitness wird dagegen eher mit gesundheitlichen Faktoren, z.B. kardiovaskulärerLeistungsfähigkeit, in Verbindung gebracht. Die aktuellen Empfehlungen finden sich in der folgenden Infobox. Grundsätzlich zeigt sich, dass Adipöse in der Regel 
einen eher inaktiven Lebensstil aufweisen. Vanhecke et al. (2009) untersuchten das Aktivitätsverhalten von 10 Personen mit einem durchschnittlichen BMI von 53,6 $\pm 11,7$ über drei Tage. Pro Tag lag der mittlere Kalorienverbrauch bei 2.668 \pm 481 Kilokalorien bzw. $3.763 \pm 2.223$ Schritten. Die untersuchten Männer $(n=4)$ und Frauen $(n=6)$ verbrachten 23 Stunden und 51,6 min am Tag körperlich inaktiv (inkl. Schlafen), das bedeutet einen Verbrauch unter 3 METs, die verbleibenden 8,4 min in moderater Aktivität ( 3 bis 6 METs). Im Rahmen der „America On the Move Study" wurden 1.921 Erwachsene mittels Pedometer untersucht. Durchschnittlich gingen sie 5.117 Schritte pro Tag, Adipöse etwa 1.500 weniger. Das allgemein empfohlene Ziel von 10.000 Schritten pro Tag wird also besonders in dieser Klientel deutlich unterschritten, sodass sich hieraus in Abhängigkeit von der Ausgangssituation Ziele vereinbaren lassen.

Empfehlungen der American Heart Association zur Steigerung von körperlicher Aktivität und Reduktion der Inaktivität, um das gesundheitliche und psychosoziale Wohlbefinden zu steigern und ein „gesundes" Körpergewicht anzustreben (mod. nach Redberg et al. 2009)

Ziel: Steigerung der körperlichen Fitness durch ein adäquates Ausdauertraining, kombiniert mit Stretching zu Steigerung der Flexibilität und Koordination und angemessenem Krafttraining zur Verbesserung der Muskelkraft

Um chronischen Erkrankungen im Erwachsenenalter vorzubeugen: zusätzlich zu den gewohnten Aktivitäten mindestens 30 min in moderater Intensität in Alltag und Freizeit an den meisten Tagen der Woche aktiv werden; mindestens aber an 5 Tagen/Woche.

Ein höherer gesundheitlicher Nutzen ergibt sich aus einer Steigerung der Dauer und/oder der Intensität.

Zum Gewichthalten bzw. zur Prävention einer übermäBigen Gewichtszunahme empfiehlt sich die Durchführung von 60 min moderater bis intensiver körperlicher Aktivität an den meisten Tagen der Woche; mindestens aber an 5 Tagen/Woche, vorausgesetzt die Kalorienzufuhr wird dem Verbrauch angepasst.

Zur Gewichtsreduktion empfiehlt sich die Durchführung von 60 bis 90 min moderater körperlicher Aktivität an den meisten Tagen der Woche; mindestens aber an 5 Tagen/Woche - vorausgesetzt die Kalorienzufuhr wird dem Verbrauch angepasst.

Ggf. sollte vor Aufnahme der körperlichen Aktivität eine (sport-)ärztliche Untersuchung zur Detektion möglicher Risiken erfolgen.
Vor dem Hintergrund von Mobilisation und Physiotherapie muss allerdings noch einmal der Begriff Sport- oder Bewegungstherapie deutlich vom Wettkampfund Leistungssport abgegrenzt werden. Im Vordergrund stehen Prozesse des Erlebens und Verhaltens, die in Gang gesetzt bzw. modifiziert werden sollen und weniger um eine Steigerung körperlicher Funktionen. Zielsetzung ist letztlich, eine die Therapie überdauernde Verhaltensänderung (funktionell, psychisch, sozial) zu erreichen (Schüle 1987; Schüle u. Deimel 1990). Inwieweit im Rahmen einer Sporttherapie stärker somatische, psychische oder soziale Zielsetzungen akzentuiert werden, hängt von der Befindlichkeit und den Bedürfnissen des einzelnen Patienten ab. Um diesem Anliegen gerecht zu werden, muss eine Vielfalt an Methoden zur Verfügung stehen, die entsprechend individueller und gruppendynamischer Aspekte, aber auch unter Berücksichtigung organisatorischer Bedingungen eingesetzt werden (Schüle 1987).

Allerdings spielt in der Klientel der morbid Adipösen die Bewegungstherapie eine untergeordnete Rolle. Der Grund ist weniger eine mangelnde Akzeptanz, als vielmehr die Vorstellung, dass die Compliance der Betroffenen falsch eingeschätzt/unterschätzt wird: Mögliche Barrieren, insbesondere gesundheitlicher Gründe (im Bereich des Bewegungsapparates), werden übersehen, und es wird vor allem verkannt, dass eine Steigerung der körperlichen Aktivität keine rasch sichtbare Gewichtsreduktion möglich macht.

Als entscheidendes Argument bleibt somit der Langzeiteffekt. Ein einstündiger Spaziergang im 0.g. Beispiel führt zwar nur zu einem Verbrauch von $300 \mathrm{kcal}$ oder einer Abnahme von $50 \mathrm{~g}$ des Körpergewichts. Wird dieser jedoch konsequent über einen Monat hinweg durchgeführt, sind dies $1,5 \mathrm{~kg}$, über ein lahr betrachtet kommt es zu einem Verlust von $18 \mathrm{~kg}$ !

Den Einstieg kann - je nach gesundheitlicher Lage die Physiotherapie bieten, denn teilweise stellen bereits Alltagsaktivitäten für diese Personengruppen hohe Intensitäten dar. In einer Cruppe amerikanischer Indianer $(n=457)$ gaben insbesondere die morbid Adipösen signifikant häufiger Bewegungseinschränkungen (51,1\%), v.a. auch Arthritiden (41,9\%) an. Aber auch Frakturen und Knochenverletzungen $(12,5 \%)$ und Herzbeschwerden $(8,3 \%)$ fanden sich in dieser Cruppe am häufigsten; nur etwa ein Fünftel empfand den Gesundheitszustand als gut (Hodge et al. 2011). Kein Unterschied bestand bei Gehbeschwerden, sodass eine Steigerung der täglichen Schrittzahl 
und der Einsatz von Schrittzählern zur Motivation auch in dieser Klientel eine gute Möglichkeit bietet, die körperliche Aktivität zu steigern. Allerdings müssen mögliche Barrieren ebenfalls berücksichtigt werden und ggf. zur Suche nach Alternativen führen. Hulens et al. (2003) zeigten, dass adipöse, insbesondere morbid adipöse Frauen häufiger an Inkontinenz, Kniebeschwerden, aber auch Rücken- und Hüftschmerzen leiden. Außerdem gingen sie langsamer und hatten häufiger Atemnot und muskuloskelettale Beschwerden am Ende des Walkings.

\subsection{Transfer in die Praxis}

Die Durchführung von Sportprogrammen für Übergewichtige stößt in der Praxis auf Probleme. Dies nicht zuletzt, weil Angebote für Betroffene mit morbider Adipositas - wenn überhaupt - nur spärlich vorhanden sind. Als weiteres praktisches Problem muss berücksichtigt werden, dass Übergewichtige sich wegen ihrer Körperfülle schämen und sich scheuen, diese in der Öffentlichkeit zu präsentieren.

Zusätzlich muss in der Ausübung die individuelle Belastbarkeit berücksichtigt werden; teilweise stellen bereits Alltagsaktivitäten für diese Personengruppen hohe Belastungen dar. Als mögliche Unterscheidung kann neben der Ergometrie beispielsweise der 6-minGehtest genutzt werden oder - für die Praxis leicht handhabbar- eine Mindestgehstrecke von z.B. 75 m. Für sehr leistungsschwache Personen kann dann der Einstieg möglicherweise nur über physiotherapeutische Maßnahmen erfolgen. Das gilt insbesondere für Personen, bei denen aufgrund ihrer Körperfülle kein Test - inkl. Ergometrie - möglich ist. Bewegungsprogramme sind dann erst nach einer Cewichtsreduktion sinnvoll, z.B. durch bariatrische Chirurgie. Allerdings kann eine geringe körperliche Belastbarkeit auch durch die Auswahl geeigneter Sportformen bzw. entsprechende Modifikationen gemeistert werden. Einem sehr stark Übergewichtigen ist es nur schwer möglich, angesichts seines Missverhältnisses zwischen aktiver Muskelmasse und Fettmasse $z u$ joggen. Besser ist es, weniger intensiv Sport zu treiben, z.B. Walking, oder die Armbewegung beim Nordic Walking besser zu nutzen, oder Sportarten zu bevorzugen, in denen das Körpergewicht getragen wird oder „gleiten“ kann, z.B. beim Radfahren oder Skilanglauf.

Besonders bewährt hat sich daher Schwimmen bzw. bei extremem Übergewicht der Aufenthalt im Wasser zumindest in Form von Wassergymnastik. Im Wasser erfährt der Übergewichtige Auftrieb, er braucht nur noch wenige Prozente seiner Körpermasse selbst zu tragen. Das Wasser kann die als unangenehm empfundene Körpermasse verdecken. Auch aus Sicht möglicher Schäden am Bewegungsapparat, z.B. Gonarthrosen ist dies günstig. Hinzu kommt, dass der Adipöse im Wasser durch seine bessere Wärmeisolation einen Vorteil mitbringt. Selbst bei extremem Übergewicht kann somit v.a. in warmem Wasser von Anfang an mit der Bewegungstherapie begonnen werden. Allerdings muss insbesondere das Schamgefühl berücksichtigt werden, daher empfiehlt sich die Nutzung von Bewegungsbädern (im stationären Bereich), mit Einzel- oder Cruppentraining von ähnlich Betroffenen.

Bei jüngeren Übergewichtigen kommen auch andere Belastungsformen infrage, in denen sie durch ihr hohes Gewicht Vorteile gegenüber Normalgewichtigen mitbringen und die daher motivierend wirken. Genannt seien Sportarten, bei denen der Übergewichtige seine Körpermasse positiv einsetzen kann, wie etwa beim Kugelstoßen oder Ringen, teilweise auch in Mannschaftssportarten, bei denen mit Körpereinsatz gearbeitet wird.

Entscheidend für die Therapietreue ist, wie in allen Programmen, eine entsprechende motivierende Führung sowie die Erfolgskontrolle und Vermittlung von Erfolgserlebnissen. Dies geschieht am besten durch den Einsatz von Bewegungstagebüchern, Schrittzählern etc. Das Sportprogramm sollte dabei dem Prinzip der Steigerung folgen, d.h. man sollte mit geringen „Dosen“ beginnen und entsprechend der zunehmenden Leistungsfähigkeit steigern.

Man kann zum Beispiel mit einem täglichen Gehtraining (Dauer 5 bis 10 Minuten; $3 \mathrm{x}$ am Tag) beginnen und versuchen, den Energieverbrauch über die Dauer und später auch die Intensität zu steigern, wenn es möglich ist, die Zielzahl stufenweise (z.B. pro Woche $10 \%$ mehr) auf 1.000 Schritte täglich zu steigern oder nach einer eingetretenen Gewichtsreduktion sogar mit Walking/Nordic Walking zu beginnen. Alternativen stellen funktionelle/gymnastische Übungen dar, die auch im Bett mit kleinen Gewichten, ggf. Therabändern durchgeführt werden. Die Einheiten können auch ohne Anwesenheit des Therapeuten durchgeführt und im Bewegungstagebuch vermerkt werden. Dies dient auch dem positiven Feedback durch die Betreuer. Die Therapietreue wird größer, wenn das Programm abwechslungsreicher gestaltet wird, z.B. durch zwischengeschaltete Einheiten von Radfahren oder Schwim- 
men. Je detaillierter das Programm ausgearbeitet ist und je häufiger die Gewichtskontrollen sind, umso größer wird der Erfolg sein.

Tumiati et al. (2008) führten ein neunmonatiges Heimtraining mit adipösen Personen nach einer Rehabilitationsmaßnahme durch. Das Training umfasste ein schriftlich angeleitetes Walking und Krafttraining und führte zu einer weiteren Abnahme des BMI und Bauchumfangs im Vergleich zur Kontrollgruppe, die nur mit den allgemeinen Bewegungsempfehlungen entlassen wurde. Der Nutzen, z.B. auch nach erfolgter bariatrischer Chirurgie, wird damit deutlich: Meist kommt es erst dann zu einer Steigerung der Leistungsfähigkeit. Zieht man aber die psychosozialen Aspekte in Betracht, sollten entsprechende Übungen stets in jede Art der Therapie und zu jedem Zeitpunkt in Anhängigkeit des individuellen Zustands eingebracht werden.

\section{Sportarten für morbid Adipöse}

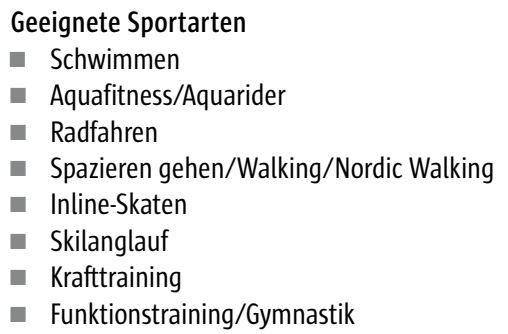

\section{Vorsicht vor}

v Sportarten mit hohen Gelenkbelastungen

\section{Zusammenfassung}

Der Nutzen von körperlicher Aktivität ist heutzutage unbestritten. Bereits eine Verbesserung der Leistungsfähigkeit stellt für Adipöse ein wichtiges prognostisch günstiges Ziel dar. Grundsätzlich gelten daher für morbid adipöse Patienten die gleichen Empfehlungen wie für alle anderen Gruppen auch; im Einzelfall muss aber entschieden werden, was Patienten (noch) an Bewegung leisten können, z.T. sind nur physiotherapeutische Übungen, Hockergymnastik, etc. möglich. Außerdem fehlen Studien, welche die Machbarkeit prüfen und damit zu evidenzbasierten Handlungsempfehlungen führen. Die aktuelle Situation, dass morbid Adipöse von Bewegungsprogrammen zumeist ausgeschlossen sind, ist sicherlich nicht zufriedenstellend.

\section{Literatur}

Bassett DR Jr, Wyatt HR, Thompson H, Peters IC, Hill JO (2010) Pedometer-measured physical activity and health behaviors in U.S. adults. Med Sci Sports Exerc 42, 1819-1825

Berg A, König D (2005) Aspekte zur Prävention und Therapie von Fettstoffwechselstörungen unter besonderer Berücksichtigung des metabolischen Syndroms. Dtsch Z Sportmed 56, 74-82

Caspersen Cl, Powell KE, Christenson GM (1985) Physical activity, exercise, and physical fitness: definitions and distinctions for health-related research. Public Health Rep 100, 126-131

Graf C (2011) Adipositas - Fit oder fett. Kardiologe 5, 51-60

Halle M, Berg A, Garwers U, Baumstark MW, Knisel W, Grathwohl D, König D, Keul I (1999) Influence of 4 weeks' intervention by exercise and diet on low-density lipoprotein subfractions in obese men with type 2 diabetes. Metabolism 48, 641-644

Hodge FS, Cantrell BG, Kim S (2011) Health status and sociodemographic characteristics of the morbidly obese American Indians. Ethn Dis 21, 52-57

Hulens M, Vansant G, Claessens AL, Lysens R, Muls E (2003) Predictors of 6-minute walk test results in lean, obese and morbidly obese women. Scand I Med Sci Sports 13, 98-105

Lee CD, Blair SN, Jackson AS (1999) Cardiorespiratory fitness, body composition, and all-cause and cardiovascular disease mortality in men. Am I Clin Nutr 69, 373-380

Lee S, Kuk JL, Katzmarzyk PT, Blair SN, Church TS, Ross R (2005) Cardiorespiratory fitness attenuates metabolic risk independent of abdominal subcutaneous and visceral fat in men. Diabetes Care 28, 895-901

Pate R, Pratt M, Blair SN, Haskell WL, Macera CA, Bouchard C, Buchner D, Ettinger W, Heath GW, King AC, Kriska A, Leon AS, Marcus BH, Morris I, Paffenbarger RS Ir, Patrick K, Pollock ML, Rippe JM, Sallis J, Wilmore JH (1995) Physical activity and public health. A recommendation from the Centers for Disease Control and Prevention and the American College of Sports Medicine. JAMA 273, 402-407

Redberg RF, Benjamin EJ, Bittner V, Braun LT, Goff DC Ir, Havas S, Labarthe DR, Limacher MC, Lloyd-jones DM, Mora S, Pearson TA, Radford MI, Smetana GW, Spertus IA, Swegler EW (2009) AHA/ ACCF 2009 performance measures for primary prevention of cardiovascular disease in adults: a report of the American College of Cardiology Foundation/American Heart Association task force on performance measures (writing committee to develop performance measures for primary prevention of cardiovascular disease): developed in collaboration with the American Academy of Family Physicians; American Association of Cardiovascular and Pulmonary Rehabilitation; and Preventive Cardiovascular Nurses Association: endorsed by the American College of Preventive Medicine, American College of Sports Medicine, and Society for Women's Health Research. Circulation 120, 12961336

Robert Koch-Institut (2005) Gesundheitsberichterstattung des Bundes. Körperliche Aktivität (Heft 26), Berlin (www.gbe-bund.de)

Schüle K (1987) Effektivität und Effizienz in der Rehabilitation. Zum Stellenwert von Bewegungstherapie und Sport. Richarz St. Augustin

Schüle K, Deimel H (1990) Gesundheitssport und Sporttherapie eine begriffliche Klärung. In: Deutscher Verband für Gesund- 
heitssport und Sporttherapie (DVGS): Gesundheitssport und Sporttherapie 6, 3

Tumiati R, Mazzoni G, Crisafulli E, Serri B, Beneventi C, Lorenzi CM, Grazzi G, Prato F, Conconi F, Fabbri LM, Clini EM (2008) Homecentred physical fitness programme in morbidly obese indi- viduals: a randomized controlled trial. Clin Rehabil 22, 940950

Vanhecke TE, Franklin BA, Miller WM, dejong AT, Coleman C), MCCullough PA (2009) Cardiorespiratory fitness and sedentary lifestyle in the morbidly obese. Clin Cardiol 32, 121-124

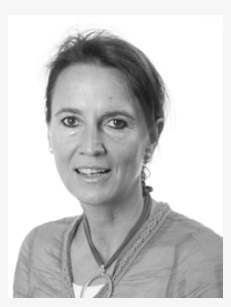

\section{Prof. Dr. med. Dr. Sportwiss. Christine Graf}

1993-1994 AIP am Martha Maria Krankenhaus Nürnberg/Innere Medizin, anschließend in der Medizinischen Klinik III (Kardiologie) der Universitätskliniken Köln tätig. Seit 1996 wissenschaftliche Mitarbeiterin am Institut für Kreislaufforschung und Sportmedizin, von August 2002 bis Mai 2008 Oberärztin am gleichen Institut. Seit Mai 2008 Leiterin der Abteilung III Bewegungs- und Gesundheitsförderung am Institut für Bewegungs- und Neurowissenschaft. Frau Professor Graf ist außerdem Vorsitzende des Sportärztebundes Nordrhein, Stellvertretende Direktorin der Akademie für Sportmedizinische Weiter- und Fortbildung und Sprecherin des Forschungsverbundes „Kölner Zentrum Prävention für Kinder und Jugendliche“ am Herzzentrum Köln (Universitätskinderkardiologie). 